Afrika Statistika

Afrika Statistika

Vol. 8, 2013, pages 491-497.

DOI: http://dx.doi.org/10.4314/afst.v8i1.1

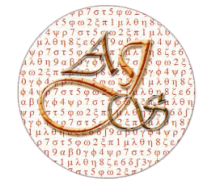

ISSN 2316-090X

\title{
On Generalized Type 1 Logistic Distribution
}

\author{
Mohammad Ahsanullah \\ Department of Management Sciences, Rider University Lawrenceville, New Jersey, 08648, USA \\ Received: October 02, 2013; Accepted: 15 November 15, 2013 \\ Copyright (c) 2013, Afrika Statistika. All rights reserved
}

\begin{abstract}
Some distributional properties of the generalized type 1 logistic distribution are given. Based on these distributional property a characterization of this distribution is presented.

Résumé. Quelques propriétés relatives à la loi de distribution de variables aléatoires suivant la loi logistique généralisée de Type 1 sont données. Nous en déduisons une caractérisation de ce type de distribution.
\end{abstract}

Key words: Conditional Expectation; Reversed Hazard Rate; Characterization.

AMS 2010 Mathematics Subject Classification : 62E10, 6215, 62E20.

\section{Introduction}

We say that an absolutely continuous random variable (rv) has a generalized type I logistic distribution $\left(G L D_{1}(\alpha)\right)$ if its cumulative distribution function $(c d f) F(x)$ is as follows:

$$
F(x)=\frac{1}{\left(1+e^{-x}\right)^{\alpha}}-\infty<x<\infty, \alpha>0 .
$$

The corresponding probability density function $(p d f)$ is given by

$$
f(x)=\frac{\alpha e^{-x}}{\left(1+e^{-x}\right)^{\alpha+1}} .
$$

The generalized logistic distribution is also called exponentiation logistic distribution. If $\alpha=1$,then generalized logistic distribution is the standard logistic distribution (SLD).The standard logistic distribution is similar in shape to normal distribution. It is symmetric around zero and bell shaped. The pdf of $G L D_{1}(\alpha)$ is an increasing function for $x<\ln \alpha$ and it is a decreasing function for $x>\ln \alpha$. It is unimodal with mode at $\ln \alpha$. It is $\log$-concave

Mohammad Ahsanullah : ahsan@rider.edu 
for all values of $\alpha$. and $X-\ln \alpha$ behaves like a type I extreme value( maximum) distribution. The Generalized logistic distribution can be either left or right skewed (when parameter $\alpha$ is less than 1 or greater than 1 respectively) or symmetric $(\alpha=1)$. GLD $(\alpha)$ has has been used in growth models and modelling of data. In this paper we will present some distributional property of $G L D_{1}(\alpha)$ and a characterization of it based on conditional expectation of $X$.

\section{Main Results}

The hazard rate of $G L D_{1}(\alpha)$ can be bathtub type or an increasing function depending on $\alpha$.The reversed hazard rate $(R H R) \tau(x)(=f(x) / F(x)$ is $\tau(x)=\alpha /(1+e)$. $\tau(x)$ is monotonically decreasing from $\infty$ to 0 as $x$ increases from $-\infty$ to $\infty$. The following is the graph of $(\tau(x) / \alpha)$.

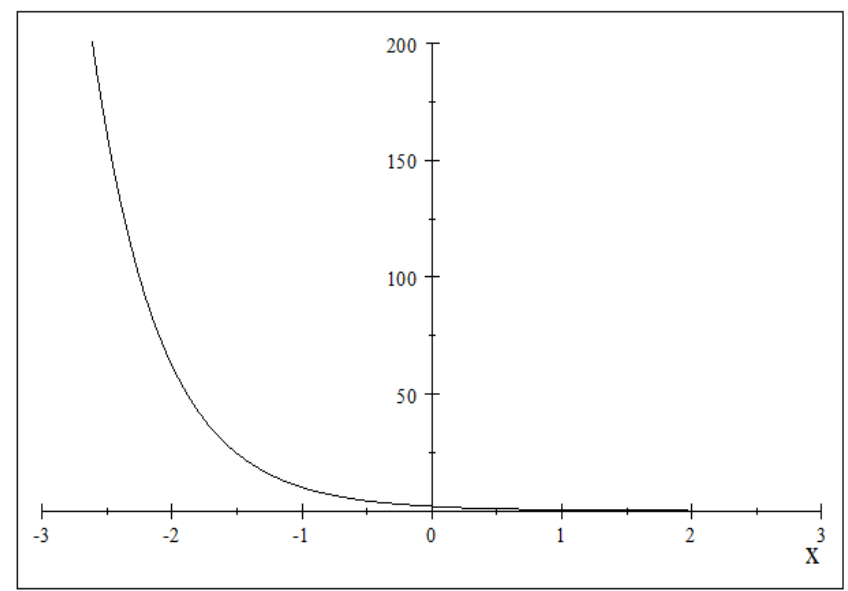

Fig. 1. $\tau(x) / \alpha$.

Suppose $Y$ is distributed as a power function distribution with $f(y)=\alpha y^{\alpha-1}, 0<y<1$, $\alpha>0$, then $X=\ln \frac{Y}{1-Y}$ is distributed as type I generalized logistic distribution. Let $\varphi(t)$ be the characteristic function of $G L D_{1}(\alpha)$, then

$$
\varphi(t)=\mathbb{E}\left(e^{i t X}\right)=\int_{-\infty}^{\infty} e^{-(1-i t) x}\left(1+e^{-x}\right)^{-(\alpha+1)} d x=\frac{\Gamma(1-i t) \Gamma(\alpha+i t)}{\Gamma(\alpha)} .
$$

We can easily calculate the mean, variance and other moments from $\varphi(t)$

$$
\mathbb{E}(X)=\Psi(\alpha)-\Psi(1)=\sum_{k=1}^{\alpha} \frac{1}{k}
$$

if $\alpha$ is an integer.

$$
\operatorname{Var}(x)=\Psi^{\prime}(x)+\Psi^{\prime}(1)
$$


M. Ahsanullah, Afrika Statistika, Vol. 8, 2013, pages 491-497. On Generalized Type 1 Logistic Distribution.

where $\Psi(x)$ and $\Psi^{\prime}(x)$ are the digamma and the polygamma function respectively. The mean is an increasing function of $\alpha$. The mean increases to $\infty$ and the variance decreases to $\Psi^{\prime}(1)\left(=\frac{\pi^{2}}{6}\right)$ as $\alpha \longrightarrow \infty$. The percentile points, $x_{p}=-\ln \left(p^{-1 / \alpha}-1\right)$ and the median, $M=-\ln \left(2^{1 / \alpha}-1\right)$. Table 1 gives the mean, median and quartile points of $G L D_{1}(1)$.

\begin{tabular}{|c|c|c|c|c|c|}
\hline$\alpha$ & Mean & first quartile & Mode & Median & Third quartile \\
\hline \hline 0.5 & -1.3863 & -2.7081 & -0.69315 & -1.0986 & 0.25131 \\
\hline 1 & 0 & -1.9086 & 0 & 0 & 1.0986 \\
\hline 5 & 2.0833 & 1.1410 & 1.6094 & 1.9058 & 2.8264 \\
\hline 10 & 2.8290 & 1.9058 & 2.3026 & 2.6342 & 3.5341 \\
\hline 15 & 3.2516 & 2.3349 & 2.7081 & 3.0514 & 3.9443 \\
\hline 25 & 3.7760 & 2.8644 & 3.2189 & 3.5715 & 4.4590 \\
\hline 50 & 4.4792 & 3.5715 & 3.9120 & 4.2716 & 5.1550 \\
\hline 100 & 5.1774 & 4.2718 & 4.6052 & 4.9683 & 5.8496 \\
\hline \hline
\end{tabular}

Table 1.

From the table we see that mean is less (greater) than median for $\alpha<1(\alpha>1)$. For $\alpha=1$, mean and median coincide. The mean is always less the the mode for all $\alpha \neq 1$. The graph of $y=\operatorname{Var}(X)$ is given in Figure 2 .

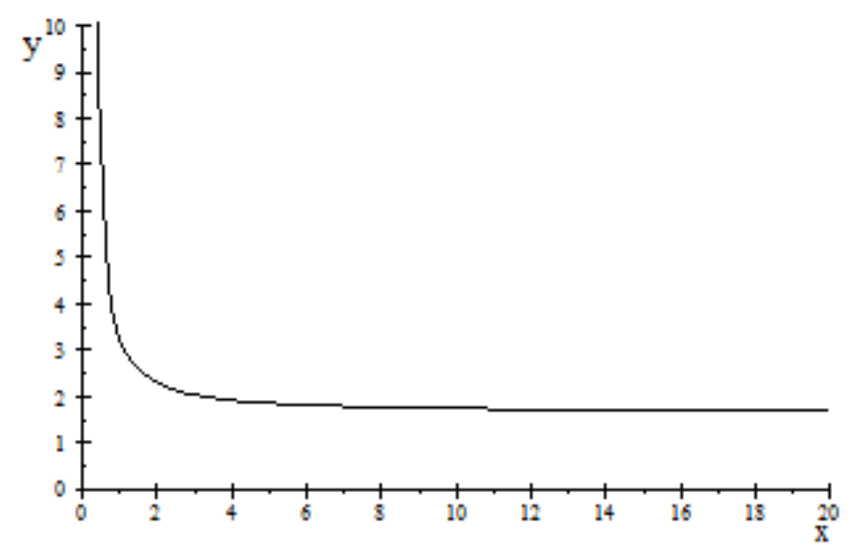

Fig. 2. $\operatorname{Var}(X)$.

From Figure 2, it is evident that $\operatorname{Var}(\mathrm{X})$ decrease to $\frac{\pi^{2}}{6}$ as $\alpha \longrightarrow \infty$.

Journal home page: www.jafristat.net 
Let $X_{1}, X_{2}, \ldots, X_{n}$ be $n$ independent and identically distributed random variables. Suppose $X_{1, n} \leq \ldots \leq X_{n, n}$ be the corresponding order statistics. Ahsanullah et al. (2013) gave some distributional properties and characterizations of logistic distribution $(G D)$. They gave the following the characteristic function, $\phi_{k, n}(t)$ (Result 1 of $X_{r, n}, 1 \leq k \leq n$ when $X$ 's are SLD. Here we give in Theorem 1 a generalization of the above result for GLD . $_{\text {. }}$

$$
\phi_{k, n}(t)=\frac{B(k+i t, n-k-i t+1}{B(k, n-k+1)},
$$

where

$$
B(p, q)=\frac{\Gamma(p) \Gamma(q)}{\Gamma(p+q)}, p . q>0 .
$$

Theorem 1. If $X$ 's are from $G D L_{1}(\alpha)$ with cdf as given in (3) and $\Psi_{k, n}(t)$ be the characteristic function of $X_{k . n}$, then.

$$
\Psi_{k, n}(t)=\frac{\alpha}{B(k, n-k+1)} \sum_{j=0}^{n-k}\left(\begin{array}{c}
n-k \\
j
\end{array}\right)(-1)^{i} B(1-i t, \alpha(n+j)+i t)
$$

Proof. The $p d f f_{k, n}(x)$ of a $k t h$ order statistics from $c d f F(x)$ and $p d f f(x)$ is (see Ahsanullah et al., 2013)

$$
f_{k, n}(x)=C_{k, n}(F(x))^{k-1}(1-F(x))^{n-k} f(x),
$$

where $C_{r, n}=n ! /(k-1) !(n-k) !$.

Substituting the pdf of $\operatorname{GLD}_{1}(\alpha)$ from (3), we obtain

$$
\begin{aligned}
\Psi_{k, n}(t) & =C_{k, n} \int_{-\infty}^{\infty} e^{i t x}\left(\frac{1}{\left(1+e^{-x}\right)^{\alpha}}\right)^{k-1}\left(1-\frac{1}{\left(1+e^{-x}\right)^{\alpha}}\right)^{n-k} \frac{\alpha e^{-x}}{\left(1+e^{-x}\right)^{\alpha+1}} d x \\
& =C_{k, n} \int_{-\infty}^{\infty} \sum_{j=0}^{n-k}(-1)^{n-k-j}\left(\begin{array}{c}
n-k \\
j
\end{array}\right) e^{i t x}\left(\frac{1}{\left(1+e^{-x}\right)^{\alpha}}\right)^{n-j} \frac{\alpha e^{-x}}{1+e^{-x}} d x \\
& =C_{k, n} \alpha \int_{0}^{1} \sum_{j=0}^{n-k}(-1)^{n-k-j}\left(\begin{array}{c}
n-k \\
j
\end{array}\right)(u)^{\alpha(n-j)+i t-1}(1-u)^{-i t} d u \\
& =\frac{\alpha}{B(k, n-k+1)} \sum_{j=0}^{n-k}\left(\begin{array}{c}
n-k \\
j
\end{array}\right)(-1)^{n-k-j} B(1-i t, \alpha(n-j)+i t) .
\end{aligned}
$$

Remark 1. For $k=n, \Psi_{n, n}(t)=\frac{\alpha B(n \alpha+i t, 1-i t)}{B(n, 1)}$. It is known (see Ahsanullah and Kirmani, 2000, page 10) that if for a continuous random variable with $c d f F(x)$, the inverse function $F^{-1}$ satisfies the condition

$$
\lim _{c \rightarrow 0} \frac{F^{-1}(1-c)-F^{-1}(1-2 c)}{F^{-1}(1-2 c)-F^{-1}(1-2 c)}=1,
$$


then $F$ belongs to the domain of attraction of the type 1 (extreme) value distribution. For $G L D_{1}(\alpha)$

$$
F^{-1}(x)=-\ln \left(x^{-1 / \alpha}-1\right)
$$

and

$$
\lim _{c \rightarrow 0} \frac{F^{-1}(1-c)-F^{-1}(1-2 c)}{F^{-1}(1-2 c)-F^{-1}(1-2 c)}=1
$$

Thus $G L D_{1}(\alpha)$ belongs to the domain of attraction of the type $I$ extreme(maximum) distribution;

$$
P\left(X_{n, n} \leq x+\ln (n \alpha)\right)=\left(\frac{1}{1+e^{-(x+n \alpha}}\right)^{n \alpha}=\left(1+\frac{e^{-x}}{n \alpha}\right)^{-n \alpha} \rightarrow e^{-e^{-x}}
$$

as $n \rightarrow \infty$.

The following theorem give a characterization of $G L D_{1}(\alpha)$.

Theorem 2. Suppose $X$ is an absolutely continuous (with respect to Lebesgue measure) random variable with cdf $F(x)$ and $p d f f(x)$. We assume that $E(X)$ exists. Then $X$ has the $G L D_{1}(\alpha)$ distribution if and only if

$$
\mathbb{E}(X \mid X \leq x)=g(x) \tau(x),
$$

where

$$
\tau(x)=\frac{f(x)}{F(x)}, g(x)=\frac{x\left(1+e^{x}\right)}{\alpha}-I_{\alpha}(x)
$$

and

$$
I_{\alpha}(x)=\frac{e^{x}\left(1+e^{-x}\right)^{\alpha+1}}{\alpha} \int_{-\infty}^{x}\left(1+e^{-u}\right)^{-\alpha} d u, \alpha>0
$$

for all $x,-\infty<x<\infty$.

To proof the Theorem, we need the following lemma.

Lemma 1. Suppose $X$ is an absolutely continuous (with respect to Lebesgue measure) random variable with cdf $F(x)$ and pdf $f(x)$. We assume that $E(X)$ exists. Then if $\mathbb{E}(X \mid X \leq x)=g(x) \tau(x)$ where $\tau(x)=f(x) / F(x)$, then

$$
f(x)=f(x)=c e^{\int \frac{x-g^{\prime}(x)}{g(x)} d x} .
$$

Proof. We have

$$
g(x)=\frac{\mathbb{E}(X \mid X \leq x)}{\tau(x)}=\frac{\int_{-\infty}^{x} u f(u) d u}{f(u)}=\frac{x F(x)}{f(x)}+\frac{\int_{-\infty}^{x} F(u) d u}{f(x)} .
$$

On simplification, we obtain from (5).

$$
x f(x)+\int_{-\infty}^{x} F(u) d u=f(x) g(x) .
$$


On differentiating both sides of (6) with respect to $x$, we have $f(x)+x f \prime(x)-f(x)=$ $f(x) g \prime(x)+f \prime(x) g(x)$ i.e.

$$
\frac{f^{\prime}(x)}{f(x)}=\frac{x-g^{\prime}(x)}{g(x)} .
$$

On integrating, we obtain from (7),

$$
f(x)=c e^{\int \frac{x-g^{\prime}(x)}{g(x)} d x}
$$

where the constant $C$ is determined by using the boundary condition $F(-\infty)=0$, and $F(\infty)=1 . \square$

\section{Proof of Theorem 2.}

Suppose that

$$
f(x)=\frac{\alpha e^{-x}}{\left(1+e^{-x}\right)^{\alpha+1}}
$$

then

$$
\begin{aligned}
g(x) & =\frac{\mathbb{E}(X \mid X \leq x)}{f(x)} \\
& =\frac{\int_{-\infty}^{x} \frac{\alpha e^{-u} u}{\left(1+e^{-u}\right)^{\alpha+1}} d u}{\frac{\alpha e^{-x}}{\left(1+e^{-x}\right)^{\alpha+1}}}=\frac{x e^{x}\left(1+e^{-x}\right)}{\alpha}-\frac{\int_{-\infty}^{x} \frac{1}{\left(1+e^{-u}\right)^{\alpha}} d u}{\frac{\alpha e^{-x}}{\left(1+e^{-x}\right)^{\alpha+1}}} \\
& =\frac{x\left(1+e^{x}\right)}{\alpha}-I_{\alpha}(x)
\end{aligned}
$$

where

$$
I(x)=\frac{e^{x}\left(1+e^{-x}\right)^{\alpha+1}}{\alpha} \int_{-\infty}^{x}\left(1+e^{-u}\right)^{-\alpha} d u^{\prime} .
$$

Suppose

$$
g(x)=\frac{x\left(1+e^{x}\right)}{\alpha}-I_{\alpha}(x)
$$

then

$$
\begin{gathered}
g \prime(x)=\frac{1+e^{-x}}{\alpha}+\frac{x e^{x}}{\alpha}-\frac{e^{x}\left(1+e^{-x}\right)^{\alpha+1}}{\alpha}\left(1+e^{-c}\right)^{-\alpha}-I_{\alpha}(x)\left\{1-(\alpha+1) \frac{-e^{-x}}{1+e-x}\right\} \\
=\frac{x e^{x}}{\alpha}+\frac{e^{x}\left(1+e^{-x}\right)^{\alpha}}{\alpha} I_{\alpha}(x)\left(1-\alpha e^{-x}\right) .
\end{gathered}
$$

We have now

$$
\begin{gathered}
x-g^{\prime}(x)=\frac{\alpha-e^{x}}{\alpha} x-I_{\alpha}(x) \frac{\alpha-e^{x}}{1+e^{-x}} \\
=\frac{\alpha-e^{x}}{1+e^{x}}\left[\frac{x\left(1+e^{x}\right)}{\alpha}-I_{\alpha}(x)\right] \\
=\frac{\alpha-e^{x}}{1+e^{x}} g(x) .
\end{gathered}
$$

Journal home page: www.jafristat.net 
M. Ahsanullah, Afrika Statistika, Vol. 8, 2013, pages 491-497. On Generalized Type 1 Logistic Distribution.

Thus

$$
\frac{f^{\prime}(x)}{f(x)}=\frac{x-g^{\prime}(x)}{g(x)}=\frac{\alpha-e^{x}}{1+e^{x}} .
$$

On integrating, we obtain from (9)

$$
f(x)=c e^{\int \frac{x-e^{x}}{1+e^{x}} d x}=c \frac{e^{-x}}{\left(1+e^{-x}\right)^{\alpha+1}} .
$$

Using the condition $\int_{-\infty}^{\infty} f(x) d x=1$, we obtain

$$
f(x)=\frac{\alpha e^{-x}}{\left(1+e^{-x}\right)^{\alpha+1}} .
$$

\section{References}

Ahsanullah, M. and Kirmani, S.N.U.A., 2008. Topics in Extreme Value. Nova Publishers, New-York, USA.

Ahsanullah, M., Nevzorov, V.B. and Shakil, M., 2013. An Introduction to Order Statistics. Atlantis Press, Paris, France.

Ahsanullah, M., Yanev, G.P and Onica, C., 2012. Characterizations of Logistic Distribution through Order Statistics with IndepndentExponential Shift. Economic Quality Control., 27. 85-96.

Lin, G.D. and Hu, C.Y., 2008. On characterizations ohe logistic distribution. J. Satatis. Plann. Inference, 138. 1147-1156. 